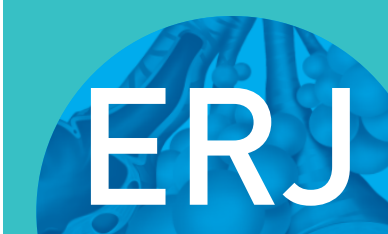

open research
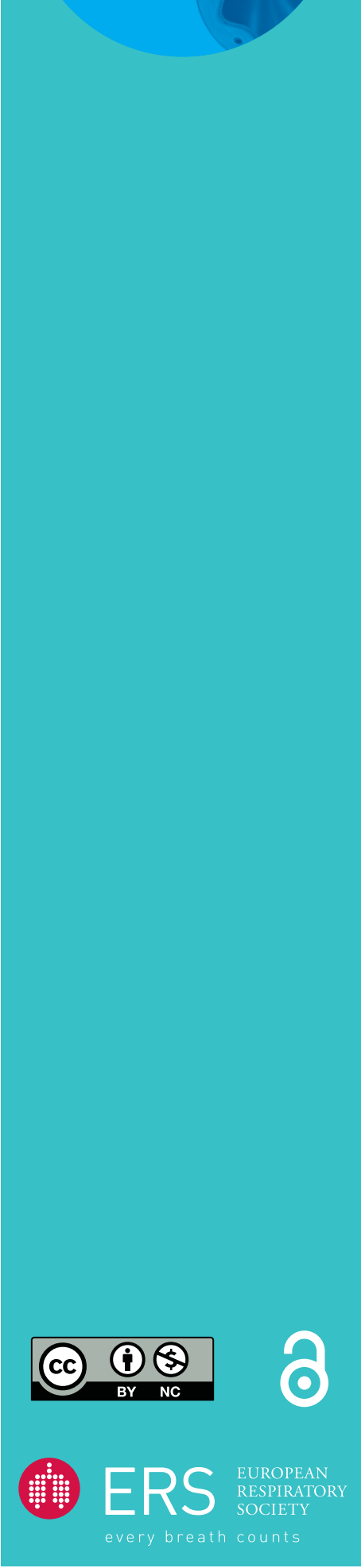

\section{Factors influencing readthrough therapy for frequent cystic fibrosis premature termination codons}

\author{
Iwona Pranke 1,2,9, Laure Bidou $3,4,9$, Natacha Martin ${ }^{5}$, Sandra Blanchet ${ }^{4}$, \\ Aurélie Hatton ${ }^{1,2}$, Sabrina Karri ${ }^{4}$, David Cornu ${ }^{4}$, Bruno Costes ${ }^{5,6}$, \\ Benoit Chevalier ${ }^{1,2}$, Danielle Tondelier ${ }^{1,2}$, Emmanuelle Girodon (1) 1,7, \\ Matthieu Coupet ${ }^{4}$, Aleksander Edelman ${ }^{1,2}$, Pascale Fanen $^{5,6,8}$, Olivier Namy ${ }^{4}$, \\ Isabelle Sermet-Gaudelus ${ }^{1,2}$ and Alexandre Hinzpeter (10 ${ }^{1,2}$
}

Affiliations: ${ }^{1}$ INSERM, U1151, Institut Necker Enfants Malades, INEM, Paris, France. ${ }^{2}$ Université Paris Descartes, Paris, France. ${ }^{3}$ Sorbonne Universités, Université Pierre et Marie Curie, UPMC, Paris, France. ${ }^{4}$ Institute for Integrative Biology of the Cell, I2BC, CEA, CNRS, Université Paris Sud, Université Paris-Saclay, Gif-sur-Yvette, France. ${ }^{5}$ INSERM, U955, Institut de Recherche Henri Mondor, Créteil, France. ${ }^{6}$ Université Paris-Est, Créteil, France. " ${ }^{7}$ aboratoire de Génétique et Biologie Moléculaires, HUPC Hôpital Cochin, AP-HP, Paris, France. ${ }^{8}$ Dept of Genetics, GH Henri Mondor, AP-HP, Créteil, France. ${ }^{9}$ Both authors contributed equally.

Correspondence: Alexandre Hinzpeter, INSERM, U1151, INEM-Institut Necker Enfants Malades, 14 Rue Maria Helena Vieira da Silva, Paris 75014, France. E-mail: alexandre.hinzpeterवinserm.fr

ABSTRACT Premature termination codons (PTCs) are generally associated with severe forms of genetic diseases. Readthrough of in-frame PTCs using small molecules is a promising therapeutic approach. Nonetheless, the outcome of preclinical studies has been low and variable. Treatment efficacy depends on: 1) the level of drug-induced readthrough, 2) the amount of target transcripts, and 3) the activity of the recoded protein. The aim of the present study was to identify, in the cystic fibrosis transmembrane conductance regulator (CFTR) model, recoded channels from readthrough therapy that may be enhanced using CFTR modulators.

First, drug-induced readthrough of 15 PTCs was measured using a dual reporter system under basal conditions and in response to gentamicin and negamycin. Secondly, exon skipping associated with these PTCs was evaluated with a minigene system. Finally, incorporated amino acids were identified by mass spectrometry and the function of the predicted recoded CFTR channels corresponding to these 15 PTCs was measured. Nonfunctional channels were subjected to CFTR-directed ivacaftor-lumacaftor treatments.

The results demonstrated that CFTR modulators increased activity of recoded channels, which could also be confirmed in cells derived from a patient.

In conclusion, this work will provide a framework to adapt treatments to the patient's genotype by identifying the most efficient molecule for each PTC and the recoded channels needing co-therapies to rescue channel function.

@ERSpublications

This study identified readthrough-recoded CFTR channels, the activity of which could be enhanced using CFTR modulators http://ow.ly/f7Gd30hBCeG

Cite this article as: Pranke I, Bidou L, Martin N, et al. Factors influencing readthrough therapy for frequent cystic fibrosis premature termination codons. ERJ Open Res 2018; 4: 00080-2017 [https:// doi.org/10.1183/23120541.00080-2017].

This article has supplementary material available from openres.ersjournals.com

Received: July 032017 | Accepted after revision: Dec 222017

Clinical trial: This study is registered at ClinicalTrials.gov with identifier number NCT02965326.

Copyright $\odot$ ERS 2018. This article is open access and distributed under the terms of the Creative Commons Attribution Non-Commercial Licence 4.0. 


\section{Introduction}

In-frame premature termination codons (PTCs) account for $\sim 11 \%$ of all described gene defects causing human genetic diseases and are often associated with a severe phenotype [1]. This is usually caused by the production of a nonfunctional truncated protein and the elimination of the PTC-carrying transcripts by the nonsense-mediated decay (NMD) pathway [2]. Additionally, PTCs can induce exon skipping more frequently than other exonic mutations [3].

Readthrough of deleterious PTCs is a promising therapeutic approach for many genetic diseases sharing this common defect. A number of small molecules have been shown to induce improper recognition of the PTC, favouring the recruitment of near-cognate tRNAs in place of the termination complex. When the PTC is in-frame, this can lead to the restoration of a functional full-length protein.

The best-characterised drugs active against PTCs are aminoglycosides. Their readthrough efficacy has been evaluated for different genes [4], including the cystic fibrosis transmembrane conductance regulator (CFTR). Different studies have demonstrated the possibility to restore a functional CFTR protein expression [5,6]. More recently, other readthrough molecules have been identified, such as PTC124 [7], RTC13 and RTC14 [8], NB54 [9] and Escin [9]. Interestingly, Escin also increased the levels of CFTR transcripts, similar to the effect of amlexanox, an NMD inhibitor that also induced readthrough [10]. Such drugs presenting dual activities increase readthrough efficacy by concomitantly increasing the amount of target transcripts.

One main factor limiting the impact of readthrough-inducing treatments is their highly variable efficacy [4]. Indeed, treatment response is influenced by the identity of the stop codon (UAG, UGA or UAA) [11], the nucleotide sequence neighbouring the PTC [12] and the amount of correctable transcripts [13]. The latter is related to the efficacy of NMD [14] and the occurrence of exon skipping [15]. Another important factor that is often overlooked is the activity of the recoded proteins. Depending on the identity of the PTC, readthrough will favour the incorporation of several near-cognate tRNAs presenting a single mismatch. Corresponding inserted amino acids have been identified by mass spectrometry in yeast [16] and more recently in mammalian expressing systems [17]. The production of full-length proteins bearing single amino acid substitutions could affect protein trafficking or function and compromise treatment efficacy.

As readthrough therapy is now under evaluation in clinical trials for cystic fibrosis (CF), it is important to predict treatment outcome and target the most responsive patients and drugs. Indeed, considering all the patients enrolled in eight different studies, around 60\% (77 out of 124) of the patients presented a partial functional restoration of CFTR activity $[5,6,18-23]$. We evaluated the main parameters influencing readthrough efficacy for the most frequent PTCs identified in CF patients, including basal and drug-induced readthrough levels, splicing effects, incorporated amino acids, and activity of the recoded proteins. We then aimed to propose a classification of CFTR PTCs with regard to their sensitivity to readthrough molecules, adapt readthrough-inducing molecules suitable to the patient's genotype and propose CFTR-directed co-therapies to increase treatment efficacy.

\section{Materials and methods \\ Constructs}

We used a dual luciferase reporter system to measure basal and drug-induced readthrough levels as previously described [24] using complementary oligonucleotides (table S1). Minigenes for CFTR exons 3, $4,11,14,15,21$ and 23 have been previously obtained by our group [25, 26]. Primers for exons 10, 12, 20, 22 and 24 are illustrated in table S10. Readthrough glutathione S-transferase (GST) proteins were produced from pYX24-GST as previously described [16]. The cDNA encoding CFTR was subcloned in the pTracer vector [27].

\section{Mutagenesis}

All mutants were generated by site-directed mutagenesis using the QuickChange XL II site-directed mutagenesis kit (Agilent, Santa Clara, CA, USA) as previously described [26].

Support statement: This study was support by the Agence Nationale de la Recherche (grant number ANR 13 BSV8 0012 02), Association Vaincre la Mucoviscidose (grant numbers RF20130500893 and RF20150501332) and Cystic Fibrosis Foundation Therapeutics (grant number 2014CFF000R-PF000100282). Funding information for this article has been deposited with the Crossref Funder Registry.

Conflict of interest: None declared. 


\section{Cell culture}

HEK293 and NIH3T3 were cultured in DMEM medium while BEAS-2B cells were cultured in LHC-8 medium, all supplemented with $10 \%$ fetal calf serum (Life Technologies, Carlsbad, CA, USA). BEAS-2B cells were transfected with TurboFect (Thermo Fisher Scientific, Waltham, MA, USA), HEK293 cells with Lipofectamin 2000 (Life Technologies) and NIH3T3 with JetPei reagent (Ozyme, St Quentin en Yvelines, France).

\section{Readthrough assay}

NIH3T3 cells were transfected and incubated for $24 \mathrm{~h}$ with gentamicin $\left(1.2 \mathrm{mg} \cdot \mathrm{mL}^{-1}\right.$; Life Technologies) or negamycin ( $2 \mathrm{mg} \cdot \mathrm{mL}^{-1}$; kindly provided by R. Matsuda (Dept of Life Sciences, Graduate School of Arts and Sciences, The University of Tokyo, Tokyo, Japan)), as previously described [24].

\section{Hybrid minigene splicing assays}

Minigenes were transfected into the BEAS-2B cells. Total RNA was purified using Trizol reagent (Life Technologies) and analysed by reverse transcriptase (RT)-PCR as previously described [26].

\section{Purification and identification of readthrough proteins by mass spectrometry}

For readthrough GST purification, the yeast strain was transformed with pYX24-GST vectors and GST was purified as previously described [16] unless the yeast was grown in the presence of $50 \mu \mathrm{g} \cdot \mathrm{mL}^{-1}$ of paromomycin (Sigma Aldrich, St Louis, MO, USA). Mass spectrometry analysis was performed on Coomassie-stained gel bands corresponding to the readthrough GST proteins. Briefly, bands were subjected to in-gel LysN digestion and readthrough peptides were further identified and quantified with a Triple-TOF 4600 mass spectrometer (AB Sciex, Warrington, UK) coupled to the nanoRSLC system (Thermo Fisher Scientific). Proteins were identified with the Mascot algorithm and an in-house database constructed by merging SwissProt with user-generated GST sequences, each harbouring one of the 20 amino acids in the readthrough site. The other search parameters were as follows: 1) digest reagent LysN (cleavage at the N-terminal of lysine); 2) cysteine carbamidomethylation, which was considered a complete modification; and 3) oxidation (methionine and tryptophan), which was considered variable. Peptide and fragment tolerances were set at $10 \mathrm{ppm}$ and $0.05 \mathrm{Da}$, respectively. Only ions with a score higher than the identity threshold and a false-positive discovery rate of $<1 \%$ (Mascot decoy option) were considered. To allow relative quantification, mass spectrometry extracted-ion chromatograms were generated for the peptides harbouring readthrough amino acids and the intensity of each chromatographic peak was corrected by a factor taking the ionisation and digestion efficiencies of each readthrough peptide into account [16].

\section{Western blot analysis}

HEK293 cells were transfected with the appropriate constructs and lysed after $48 \mathrm{~h}$. Proteins were analysed by Western blot using the mm13-4 anti-CFTR antibody (Millipore, Burlington, MA, USA), as previously described [26]. When indicated, cells were incubated for $24 \mathrm{~h}$ with $3 \mu \mathrm{M}$ VX809 (Selleckchem, Houston, TX, USA) prior to lysis.

\section{YFP-based functional assay}

CFTR activity was measured in transiently transfected HEK293 cells using the halide-sensitive yellow fluorescent protein YFP-H148Q/I152L (kindly provided by L.J. Galietta; Telethon Institute of Genetics and Medicine (TIGEM), Naples, Italy). Transfected cells were incubated for $30 \mathrm{~min}$ with $50 \mu \mathrm{L}$ of PBS containing CPT-AMPc (100 $\mu$ M; Sigma Aldrich) with and without VX770 (10 $\mu$ M; Selleckchem), and cell fluorescence was continuously measured before and after addition of $100 \mu \mathrm{L}$ of PBS-NaI with a TriStar (Berthold Technologies, Bad Wildbad, Germany). When indicated, cells were incubated for $24 \mathrm{~h}$ with VX809 $(3 \mu \mathrm{M})$ prior to measurements. Maximal slopes were converted to rates of change in intracellular iodide concentration (in $\mathrm{mM} \cdot \mathrm{s}^{-1}$ ).

\section{Primary cell culture and short-circuit current measurements}

Nasal epithelial cells from a p.Gly542*/p.Gly542* patient were obtained by nasal brushing as previously published [28] (ClinicalTrials.gov identifier NCT02965326; the study was approved by the Ile de France 2 Ethics Committee, and written informed consent was obtained (AFSSAPS (ANSM) B1005423-40, no. Eudract 2010-A00392-37; CPP IDF2: 2010-05-03-3)). Cells were amplified and conditionally reprogrammed before being seeded onto microporous filters $\left(0.33 \mathrm{~cm}^{2}\right.$ Transwell; Corning, New York, NY, USA). Once seeded on filters, cells were cultured on an air-liquid interface and re-differentiated [29]. When the transepithelial resistance of reconstituted human nasal epithelial cell culture was $>600 \Omega \cdot \mathrm{cm}^{2}$, cell cultures were incubated for $48 \mathrm{~h}$ with drugs added to the basal side. Cells were treated for $48 \mathrm{~h}$ with dimethyl sulfoxide (DMSO) vehicle, or gentamicin $\left(1 \mathrm{mg} \cdot \mathrm{mL}^{-1}\right)$ in the presence or absence of VX809 $(3 \mu \mathrm{M})$. Drugs were renewed every day of treatment. 
Short-circuit current was measured under voltage clamp conditions as previously described [28]. Transwell culture inserts were mounted in Ussing chambers (Physiologic Instruments, San Diego, CA, USA). During continuous recording of short-circuit current (in voltage clamp mode), the following inhibitors and activators were added at the apical side after stabilisation of baseline short-circuit current: sodium channel blocker amiloride (100 $\mu \mathrm{M}$; Sigma Aldrich), to inhibit the apical epithelial sodium channel (ENaC); cAMP agonist forskolin (10 $\mu \mathrm{M}$; Sigma Aldrich) and 3-isobutyl-1-methylxanthine (IBMx; $100 \mu \mathrm{M})$, to activate the transepithelial cAMP-dependent current (including chloride transport through CFTR channels); and CFTR inhibitor Inh172 ( $5 \mu \mathrm{M}$; Sigma Aldrich). The response to forskolin/IBMx inhibited by Inh172 served as an index of CFTR function.

\section{Statistics}

Quantitative variables were described as mean \pm SEM. Comparisons to wild-type (WT) conditions were made with one-way ANOVA followed by the Fisher test for p-value evaluation. For dual reporter, minigene and CFTR treatment analysis, unpaired t-tests were used to calculate significance.

\section{Results}

\section{Drug-induced readthrough levels}

Basal and drug-induced readthrough levels associated with 15 CFTR PTCs (listed in table S1) were measured in vitro. PTCs were selected based on their position and frequency in the CF population, as referred to in the CFTR2 database (www.cftr2.org). These 15 PTCs represent $86 \%$ of the CF alleles containing a stop codon (8368 occurrences out of 9745). Basal readthrough was variable, ranging from $0.01 \%$ to $0.52 \%$ (figure 1a and table S2). Gentamicin significantly increased readthrough to levels ranging from $0.12 \%$ to $2.70 \%$. Results were similar in a larger set of 29 PTCs, where three PTCs were found not to respond to treatment (figure S1a and table S2).

This response was compared to that obtained with negamycin, a structurally different molecule known to promote readthrough [30]. Results indicated that negamycin's action did not totally overlap that of gentamicin (figure 1b, figure S1b and table S2). Negamycin was less efficient than gentamicin for 20 out of 29 PTCs, including 10 that did not respond to the treatment (figure 1b, figure S1b and table S2). The two treatments had a similar effect for five sequences (p.Ser466*, p.Arg785*, p.Trp846*UAG, p.Trp846*UGA and p.Gln1268*), while negamycin outperformed gentamicin for four PTCs, namely p.Ser434*, p.Arg709*, p.Glu1104* and p.Arg1158* (figure 1, figure S1 and table S2). Finally, two PTCs did not respond to either treatment, namely p.Glu831* and p.Gln1390*.

\section{PTC-associated exon skipping}

Occurrence of splicing defects associated with these 15 CFTR PTCs was investigated using minigenes (figure 2 and table S3).

Results obtained with WT minigenes revealed the production of multiple transcripts for exons 14 and 22 (figure S2a). These additional transcripts were due to the use of two cryptic acceptor sites within exon 14 and one cryptic donor site in exon 22 (figure S2b), as assessed by sequencing of the RT-PCR products and as previously described using different minigene systems [31] and in primary cells [32]. For these minigenes, only skipping of the complete exon was considered.

While no additional isoforms were detected, significant increased exon skipping, ranging from $6 \%$ up to $44 \%$, was observed for four out of the 15 selected PTCs, namely p.Glu60*, p.Arg553*, p.Arg785* and p.Trp $846^{\star}$ UGA (figure 2 and table S3).

\section{Identification of incorporated amino acids upon aminoglycoside treatment}

We based the recoding rules on our previous study performed under basal conditions in yeast (table S4) [16]. To further validate these results, cells were incubated with the aminoglycoside paromomycin, which is less toxic for yeast compared with gentamicin or G418. The amino acids inserted were identified and quantified by mass spectrometry (figure $3 \mathrm{a}-\mathrm{c}$ and figure S3). Results indicated that the aminoglycoside slightly modified the ratio of incorporated amino acids (figure 3d). Interestingly, no new amino acid was identified in presence of the aminoglycoside compared with control conditions (figure S3 and table S4). These results are in good agreement with data from two recently published studies $[17,33]$ obtained in the presence of G418 (another aminoglycoside).

According to the amino acid incorporated, recoding of the 15 selected PTCs would lead to the production of either WT proteins or protein variants (figure 4 and table S5; topology based on [34]). For most of the protein variants, we only considered the major recoded amino acid. For the frequent p.Tyr122*, p.Gly542* and p.Trp $1282^{\star}$ mutations, both major and minor recoded amino acids were considered. The p.Trp $1282^{\star}$ mutation was not studied as the major recoded protein corresponded to the WT protein, while the minor 

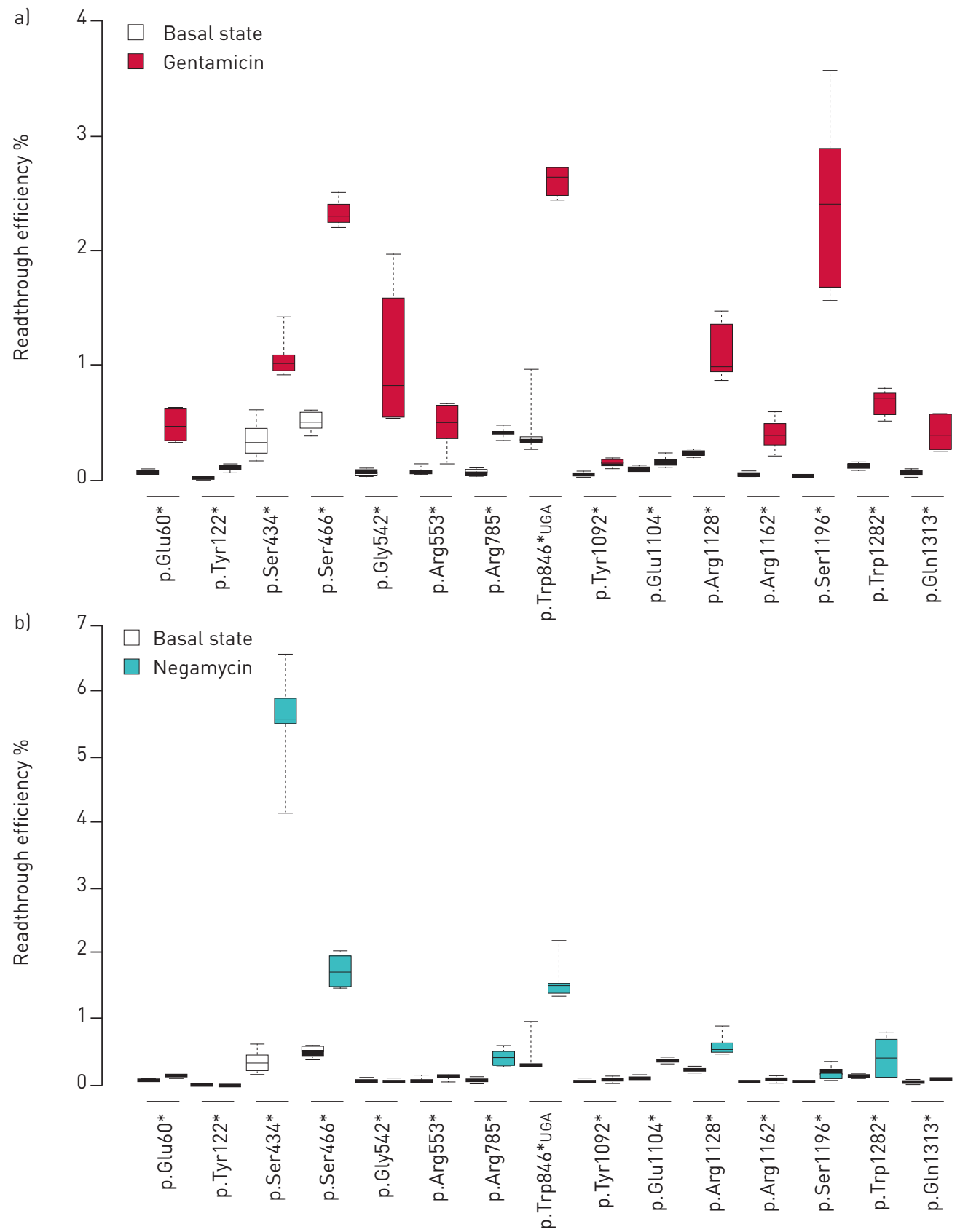

FIGURE 1 Readthrough levels measured for the indicated cystic fibrosis transmembrane conductance regulator (CFTR) premature termination codon mutations, at basal state and after incubation with a) gentamicin or b) negamycin. Central lines show the medians; box limits indicate the 25th and 75th percentiles as determined by R software; whiskers extend 1.5 times the interquartile range from the 25th and 75th percentiles; $n>5$ for all samples.

recoded proteins (p.Trp1282Arg and p.Trp1282Cys) were known CF-causing mutations leading to nonfunctional channels (CFTR1 Cystic Fibrosis Mutation Database; www.genet.sickkids.on.ca/PicturePage. html). Therefore, 17 protein variants were selected to be characterised, as five are predicted to be recoded to WT (figure 4 and table S5).

\section{Function of the predicted recoded channels}

CFTR mutants were first characterised by Western blot. Analysis of CFTR revealed both fully glycosylated (band C) and core glycosylated (band B) CFTR (figure 5a). The $\mathrm{C} /(\mathrm{B}+\mathrm{C})$ ratio, a commonly used hallmark to assess CFTR maturation, was $91 \pm 1.5 \%(\mathrm{n}=10)$ for WT CFTR, while it was reduced to $19 \pm 1.4 \%(\mathrm{n}=9$; $\mathrm{p}<0.0001$ ) for the maturation-defective p.Phe508del channel (figure 5b and table S6). Five proteins 


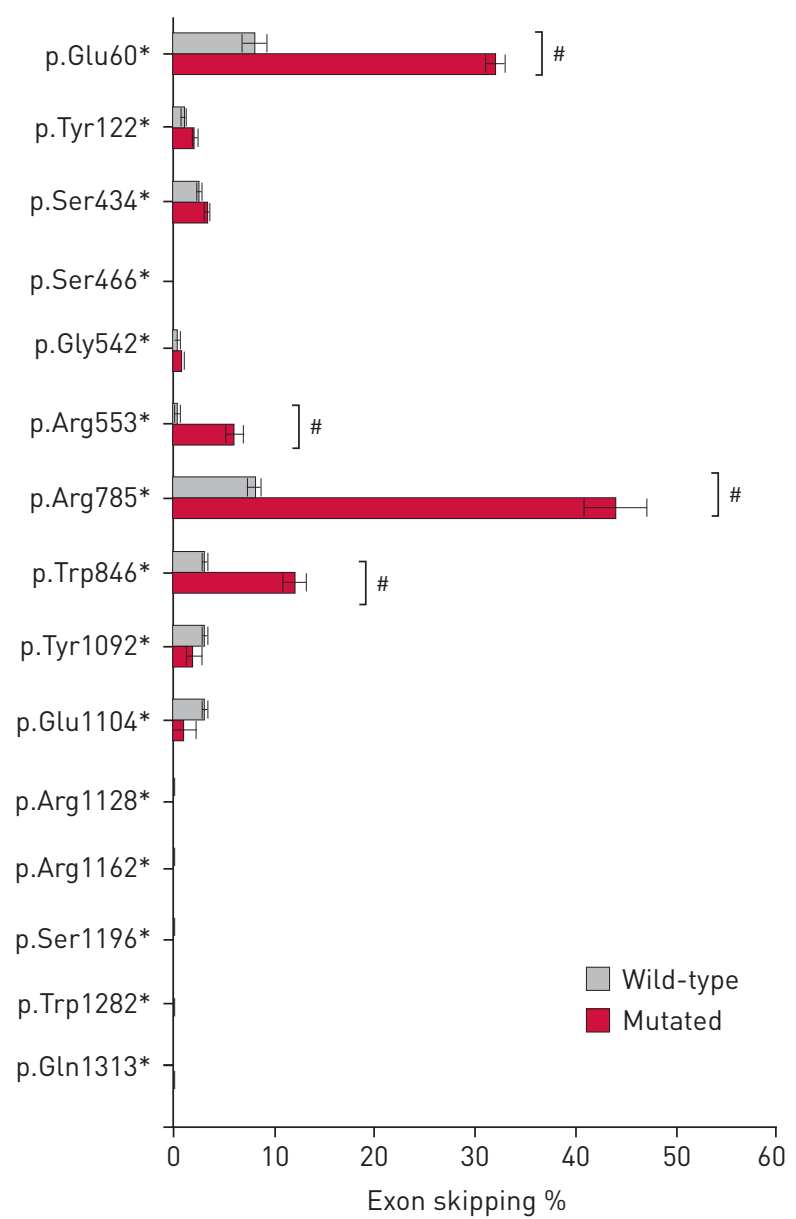

FIGURE 2 In vitro quantification of exon skipping associated with 15 premature termination codons using minigenes. The percentage of exon skipping measured by reverse transcriptase-PCR with minigenes containing wild-type exons or indicated mutated exons is shown. \#: significant increased exon skipping ( $p<0.001$, unpaired t-test, $n=5-10$ ).

presented a maturation defect, either similar to p.Phe508del (p.Glu60Tyr and p.Ser466Trp) or partial (p.Gly542Trp, p.Arg553Trp and p.Glu1104Tyr) (figure 5 and table S6).

The function of the CFTR mutants was then measured using a halide-sensitive YFP-based assay. Measurements revealed an iodide transport of $0.04 \pm 0.01 \mathrm{mM} \cdot \mathrm{s}^{-1}(\mathrm{n}=20)$ in mock-transfected HEK293 cells, a level similar to p.Phe508del-transfected cells $\left(0.04 \pm 0.01 \mathrm{mM} \cdot \mathrm{s}^{-1} ; \mathrm{n}=10\right)$ and significantly lower than that of WT-transfected cells $\left(0.27 \pm 0.02 \mathrm{mM} \cdot \mathrm{s}^{-1} ; \mathrm{p}<0.0001 ; \mathrm{n}=14\right)$ (figure $6 \mathrm{a}$ and table S7). 10 channels had an activity comparable to WT channels, while seven had a significantly reduced transport rate (figure $6 \mathrm{~b}$ and table S7). In a second set of experiments, cells were incubated with the CFTR potentiator VX770. Mock-transfected or p.Phe508del-expressing cells were not affected by the treatment, whereas WT-expressing cells showed a 1.8 -fold increase of the iodide transport rate $\left(0.52 \pm 0.05 \mathrm{mM} \cdot \mathrm{s}^{-1} ; \mathrm{n}=10\right.$; $\mathrm{p}<0.0001$ ) (figure $6 \mathrm{~b}$ and table S7). A similar increase was observed with the 10 channels presenting a normal basal activity. For channels presenting a reduced amount of band C (p.Gly542Trp, p.Arg553Trp and p.Glu1104Tyr), the potentiator significantly enhanced channel function, while treatment had no effect on maturation-defective channels p.Glu60Tyr and p.Ser466Trp (figure 6b and table S7).

Proteins presenting both maturation and functional defects were incubated with the CFTR corrector VX809, a molecule developed to correct the maturation deficiency of p.Phe508del. Results indicated an increased maturation for all treated proteins (figure $7 \mathrm{a}$ and $\mathrm{b}$ and table S6) that was concordantly associated with an increase in channel activity, in response to the VX809/VX770 combination (figure 7c and tables S6 and S7). Plotting the transport rates with the corresponding maturation ratio of CFTR (figure S4) revealed that mutants clustered into two groups under basal conditions. The first group presented a reduced maturation and channel activity (p.Gly542Trp, p.Arg553Trp and p.Glu1104Tyr), while the second group contained channels presenting a defective maturation and function (p.Glu60Tyr and p.Ser466Trp), similar to p.Phe508del. Interestingly, all constructs treated with the VX809/VX770 

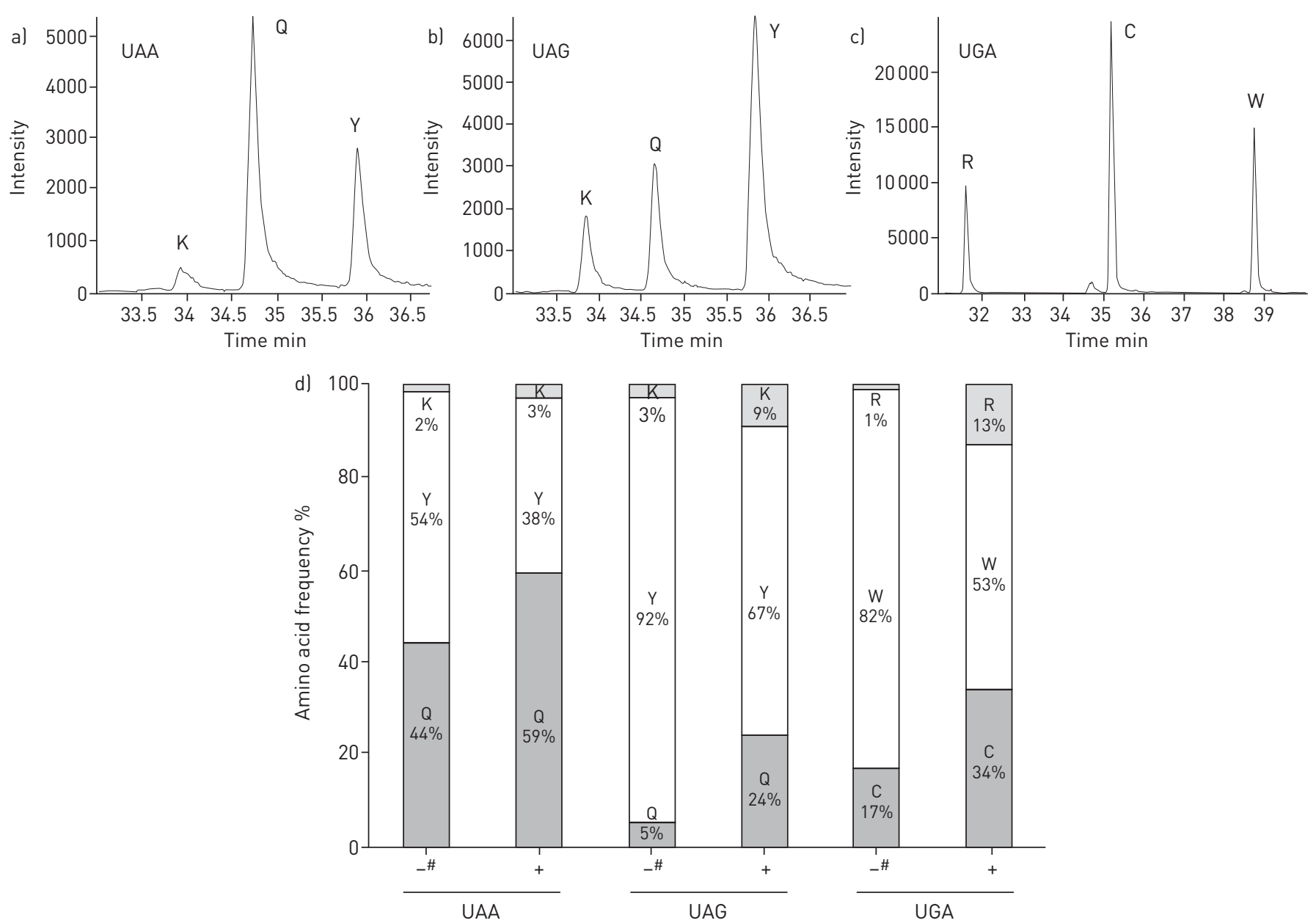

FIGURE 3 Quantification of readthrough peptides in the presence of paromomycin. a-c) Mass spectrometry extracted-ion chromatograms of readthrough peptides for UAA, UAG and UGA stop codons. d) Relative frequencies of the readthrough amino acids incorporated at UAA, UAG and UGA in the presence $(+)$ and absence $(-)$ of paromomycin. Quantifications were performed as described in the Materials and methods section. \#: data previously obtained by BLANCHET et al. [16] in the same conditions.

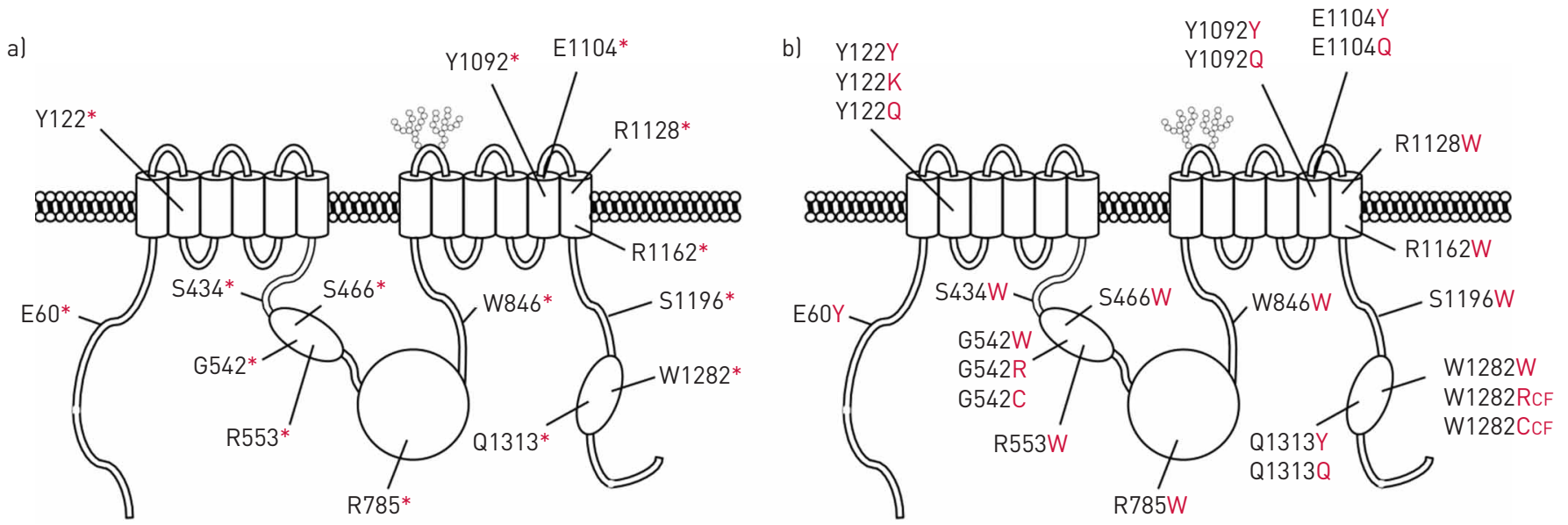

FIGURE 4 Representation of the 15 selected premature termination codons (PTCs) and their corresponding recoded major protein. Position of a) the 15 selected PTCs and b) corresponding recoded major amino acids. For the most studied p.Tyr122*, p.Gly542*, and p.Trp1282*, both major and minor recoded proteins are indicated. CF: known cystic fibrosis-causing mutations. 

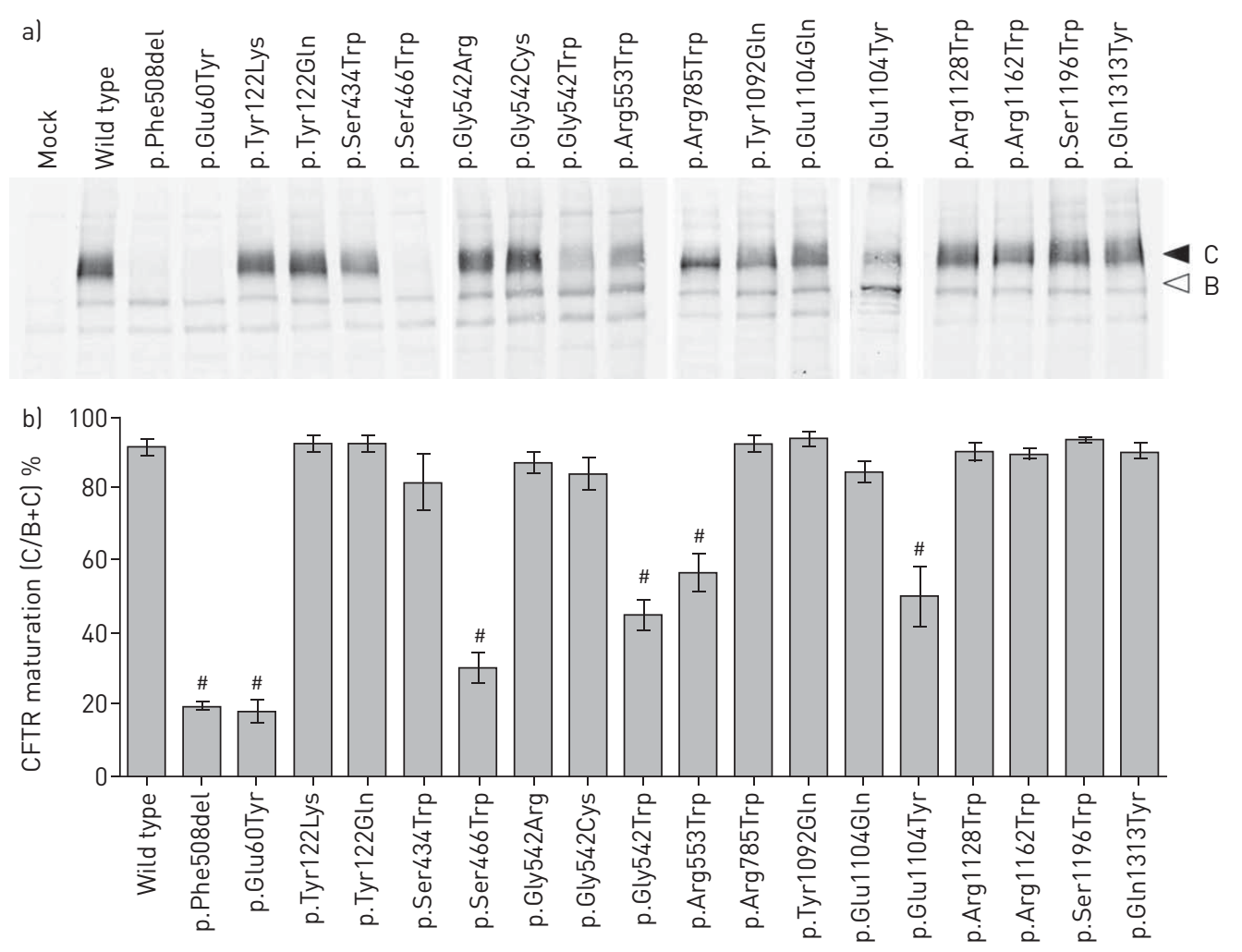

FIGURE 5 Maturation of recoded cystic fibrosis transmembrane conductance regulator (CFTR) channels a) Representative Western blot obtained from HEK293 cells expressing the indicated construct. CFTR bands $C$ and $B$ are indicated. b) CFTR maturation as measured using the $C /(B+C)$ ratio under control conditions. \#: significant difference compared with wild-type control conditions ( $p<0.01$, ANOVA followed by a Fisher test).

combination clustered in a single group independently of their untreated basal level, reaching around $70-80 \%$ of control WT function and normal maturation (figure S4 and tables S6 and S7). Correction of p.Phe508del reached around 50\% of WT function and maturation, which could reflect additional defects such as reduced cell surface stability and channel activity. A strong correlation between maturation ratio and channel function was obtained for the set of studied CFTR mutants $\left(\mathrm{R}^{2}=0.83\right)$ (figure S4).

\section{Measure of readthrough therapy efficiency in primary epithelial cells}

Primary human nasal epithelial cells were sampled by nasal brushing from a p.Gly $542 * / p . G l y 542^{\star}$ patient and cultivated on an air-liquid interface. Cells were treated for $48 \mathrm{~h}$ with DMSO vehicle, or gentamicin $\left(1 \mathrm{mg} \cdot \mathrm{mL}^{-1}\right)$ with or without VX809 $(3 \mu \mathrm{M})$. Compared with DMSO-treated cell cultures, a detectable increase of CFTR-dependent chloride secretion was observed by short-circuit current measurements after gentamicin treatment, which was much enhanced in the presence of VX809 (figure 7d-f).

\section{Global classification of PTCs with regard to readthrough therapy}

Readthrough treatment efficacy was predicted by combining the level of drug-induced readthrough, the occurrence of exon skipping and the function of the predicted recoded channel, as illustrated in table 1 and table S8.

To evaluate the relative readthrough stimulation, PTCs were classified with regard to their response to gentamicin (figure S5a) or negamycin (figure S5b). This representation revealed that the response could be considered as either high (readthrough $>1.5 \%$ ), moderate (readthrough $>0.5 \%$ and $<1.5 \%$ ) or low (readthrough $<0.5 \%$ ). The minigene assay discriminated between mutations that reduced the amount of correctable transcripts (low levels of transcripts) and those having no effect (high levels of transcripts). Finally, channel function corresponding to the major recoded protein was considered as normal (high; $>80 \%$ of WT), reduced (moderate; $20-80 \%$ of WT) or defective (low; $<20 \%$ of WT). For the UAA stop codons, which are recoded into two major channels (-Tyr and -Gln), the function was considered as reduced if one channel was defective. 

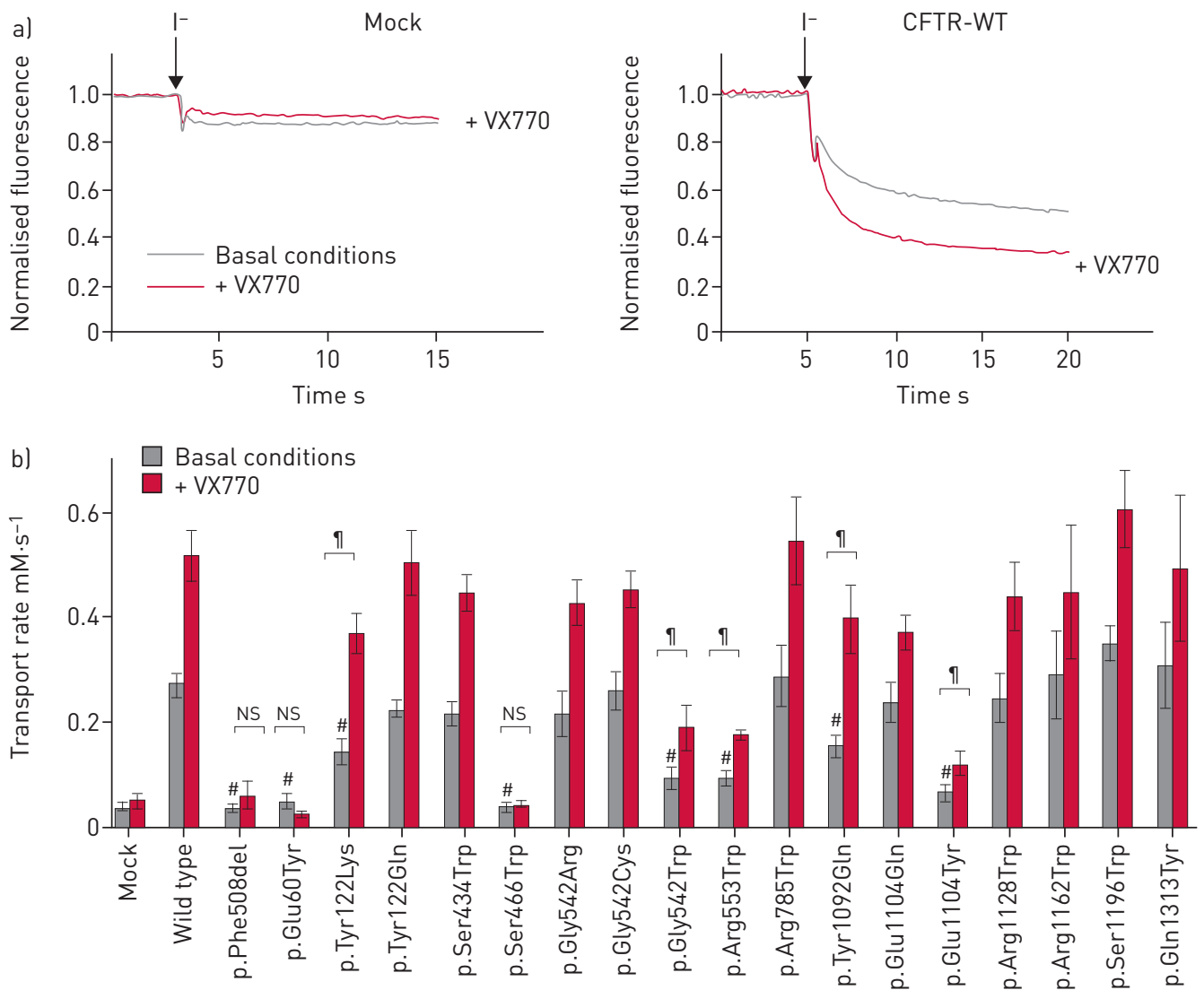

FIGURE 6 Function of recoded cystic fibrosis transmembrane conductance regulator (CFTR) channels. a) Representative recordings obtained from HEK293 cells expressing the indicated construct (mock or CFTR-WT) before and after addition of iodide-containing PBS (injection indicated with an arrow), and with and without the CFTR potentiator VX770. b) Transport rates measured under basal conditions and in the presence of the CFTR potentiator VX770. \#: significant difference compared with wild-type control conditions ( $p<0.01$, ANOVA followed by a Fisher test); ": significant effect of VX770 ( $p<0.01$, unpaired t-test); NS: nonsignificant effect of VX770.

The levels of these individual skills were then combined to assess overall treatment response (low, moderate or high), with each defect considered as affecting the global response equally. This simple matrix enabled the proposal of a classification of predicted readthrough. 10 out of 15 PTCs were predicted to have a low response to readthrough induced by gentamicin and four to have a moderate response, while one had a high response, namely p.Ser $1196^{*}$. The response to negamycin was expected to be low for 14 out of 15 PTCs and globally lower than with gentamicin. Nonetheless, p.Ser434* was predicted to respond better with negamycin (table S8). Importantly, all CFTR PTCs would benefit from additional co-therapies. Indeed, the activity of all the tested channels was enhanced by the VX770 potentiator, while mutants presenting a trafficking defect benefited from a corrector such as VX809.

Classification was supported by preclinical studies, which showed that patients carrying PTCs predicted to be moderately or highly responsive to treatment responded on average with a higher frequency compared to PTCs predicted to be low responders (table 1). Therefore, the proposed classification can explain part of the variability observed in these eight studies.

\section{Discussion}

This study provides the first extensive study to assess and predict the efficacy of readthrough-inducing molecules for a panel of frequent disease-causing PTCs in the CFTR gene. It measured basal and drug-induced readthrough, identified PTC-associated splicing defects and evaluated the function of predicted recoded channels. The combination of these results enabled us to adapt the readthrough-inducing treatment to the patient's genotype and propose CFTR-directed co-therapies to increase efficacy.

Readthrough efficacy depends on both the identity of the PTC (with the following sequence: $\mathrm{UAA}<\mathrm{UAG}<\mathrm{UGA}$ ) $[7,35]$ and the local nucleotide sequence (with a positive effect of a uracil in position -1 
a)

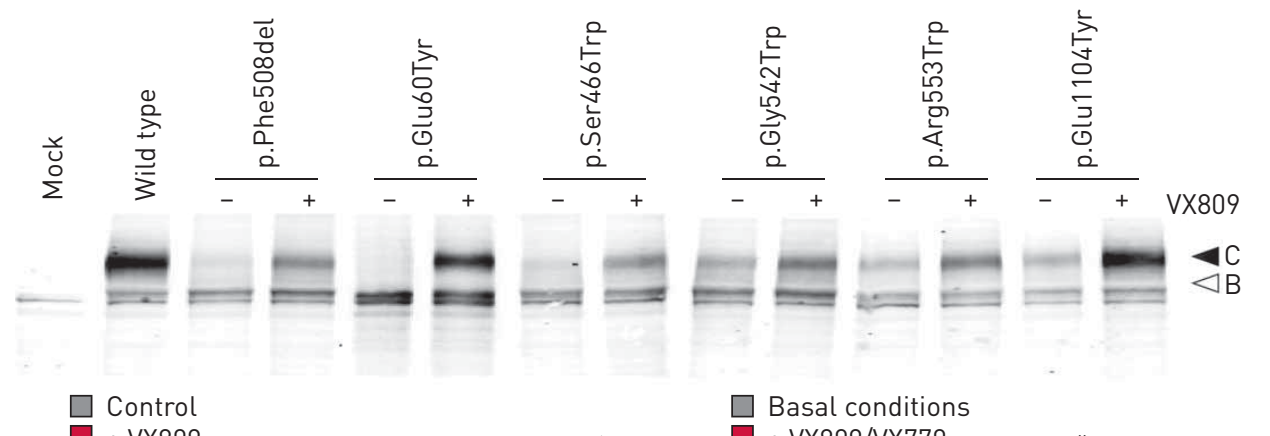

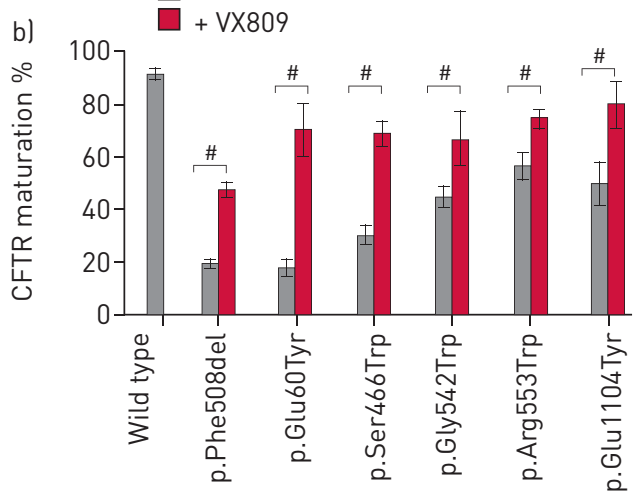

d)

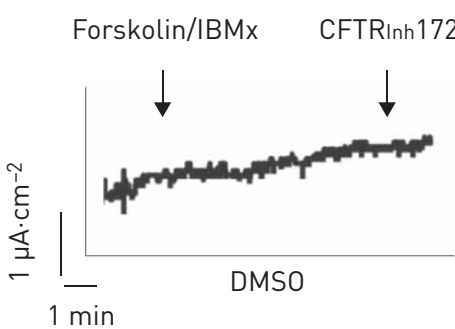

e)

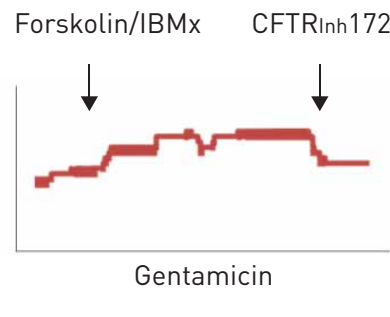

c)

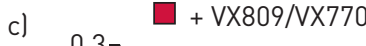

FIGURE 7 Rescue of misfolded recoded cystic fibrosis transmembrane conductance regulator (CFTR) channels. a) Representative Western blot obtained from HEK293 cells expressing the indicated mutation under basal conditions or after VX809 treatment $(3 \mu \mathrm{M}, 24 \mathrm{~h})$. CFTR bands C and B are indicated. b) CFTR maturation of the indicated construct as measured using the $C /(B+C)$ ratio under control conditions and after incubation with the corrector VX809. c) Transport rates measured under basal conditions and in presence of the corrector/potentiator combination (VX809/VX770). \#: significant effect of treatment ( $p<0.01$, unpaired t-test). d-f) Short-circuit current measurements of primary nasal epithelial cells (p.Gly542*/p.Gly542*) treated with d) dimethyl sulfoxide (DMSO) vehicle, e) gentamicin at a concentration of $1 \mathrm{mg} \cdot \mathrm{mL}^{-1}$ for 2 days, or f) gentamicin $\left(1 \mathrm{mg} \cdot \mathrm{mL}^{-1}\right)$ with VX809 $(3 \mu \mathrm{M})$. IBMx: 3-isobutyl-1-methylxanthine; CFTRInh172: CFTR inhibitor.

and a cytidine in position +4) [24]. Our results are in accordance with these previous observations as out of the 29 PTCs tested, UGA represented nine out of the 10 best responders and two of the four highest responders had the optimal U UGA C sequence. Readthrough was also induced with negamycin and, interestingly, responses were not overlapping. Indeed, even if negamycin induced lower levels of readthrough compared to gentamicin for 20 out of the 29 PTCs tested, it outperformed gentamicin for four. For these PTCs, negamycin would therefore be more adapted, raising the possibility of screening different readthrough-inducing drugs to identify the most efficient molecule for each patient's genotype.

The amount of target transcripts also directly influences readthrough efficacy; hence, a positive correlation has been obtained between chloride transport and the level of CFTR transcript after gentamicin nasal application [13]. This amount is mainly dependent on two parameters: 1) the level of NMD [13] and 2) the occurrence of a splicing defect [15]. Therefore, transcripts targeted by readthrough therapy should be quantified when mRNA from a relevant tissue is available, especially as NMD was found to be variable from one individual to another, even for the same mutation. To date, only a fraction of the 165 PTCs identified in CFTR have been studied for their ability to alter exon recognition $[26,36]$. In this study, we used minigenes to identify increased exon skipping for four out of 15 PTCs tested. These data will need to be confirmed in native tissues. 
TABLE 1 Predicted readthrough response to gentamicin and preclinical studies performed on cystic fibrosis patients carrying premature termination codons (PTCs)

\begin{tabular}{|c|c|c|c|c|c|c|c|c|}
\hline \multirow[t]{2}{*}{ Mutation } & \multicolumn{5}{|c|}{ In vitro assay } & \multicolumn{3}{|c|}{ Preclinical studies } \\
\hline & $\begin{array}{l}\text { Readthrough } \\
\text { gentamicin }\end{array}$ & $\begin{array}{l}\text { Target mRNA } \\
\text { (exon } \\
\text { skipping) }\end{array}$ & Function ${ }^{\#}$ & $\begin{array}{l}\text { Overall } \\
\text { response }^{\pi}\end{array}$ & $\begin{array}{l}\text { Additional } \\
\text { CFTR-directed } \\
\text { treatments }\end{array}$ & $\begin{array}{l}\text { Readthrough } \\
\text { gentamicin }^{+}\end{array}$ & $\begin{array}{l}\text { Readthrough } \\
\text { PTC124 }^{+}\end{array}$ & Response \\
\hline p.Glu60* & Low & Low & Low & Low & $\begin{array}{l}\text { Potentiator } \\
+ \text { corrector }\end{array}$ & 0/1 [21] & & $0 / 1(0 \%)$ \\
\hline p.Tyr122* & Low & High & High & Low & Potentiator & $6 / 9$ [5] & 8/14 [19] & $\begin{array}{l}14 / 23 \\
(60 \%)\end{array}$ \\
\hline p.Ser434* & Moderate & High & High & Moderate & Potentiator & & & \\
\hline p.Ser466*UGA & High & High & Low & Low & $\begin{array}{l}\text { Potentiator } \\
\text { +corrector }\end{array}$ & & & \\
\hline p.Gly542* & Moderate & High & Moderate & Moderate & $\begin{array}{l}\text { Potentiator } \\
\text { +corrector }\end{array}$ & $\begin{array}{l}0 / 1[5] \\
0 / 4[21] \\
0 / 3[20]\end{array}$ & $\begin{array}{l}4 / 4[22] \\
2 / 3[18] \\
8 / 14[19]\end{array}$ & $\begin{array}{l}14 / 29 \\
(48 \%)\end{array}$ \\
\hline p.Arg785* & Low & Low & High & Low & Potentiator & & & \\
\hline p.Trp846*UGA & High & Low & High & Low & Potentiator & & 0/1 [19] & $0 / 1(0 \%)$ \\
\hline p.Tyr1092*UAG & Low & High & Moderate & Low & Potentiator & $0 / 1[21]$ & & $0 / 1(0 \%)$ \\
\hline p.Glu1104* & Low & High & Moderate & Low & $\begin{array}{l}\text { Potentiator } \\
\text { +corrector }\end{array}$ & & $1 / 2[19]$ & $1 / 2(50 \%)$ \\
\hline p.Arg1128* & Moderate & High & High & Moderate & Potentiator & & & \\
\hline p.Arg1162* & Low & High & High & Low & Potentiator & $\begin{array}{l}0 / 1[5] \\
0 / 1[21]\end{array}$ & $1 / 2[19]$ & $1 / 4(25 \%)$ \\
\hline p.Ser1196* & High & High & High & High & Potentiator & & & \\
\hline p.Trp1282* & Moderate & High & High & Moderate & Potentiator & $\begin{array}{c}4 / 6[23] \\
7 / 9[13] \\
0 / 1[5] \\
0 / 1[21] \\
0 / 1[20]\end{array}$ & $\begin{array}{c}12 / 17[22] \\
8 / 14[18] \\
2 / 4[19]\end{array}$ & $\begin{array}{l}33 / 53 \\
(62 \%)\end{array}$ \\
\hline
\end{tabular}

Translational suppression of PTCs by readthrough will lead to the production of a pool of proteins containing potential protein variants. This has been confirmed by the identification of the incorporated amino acid by mass spectrometry under basal conditions [16] and under aminoglycoside treatment (figure $3 \mathrm{~d}$ and table S4). Interestingly, no new amino acid was found at the stop codon position in the presence of paromomycin (figure S3). This clearly indicates that aminoglycosides promote stop codon readthrough by increasing the incorporation of near-cognate tRNAs that are already naturally incorporated in the absence of the drug and not by promoting the insertion of a new tRNA. A recent structural study showed that gentamicin and paromomycin bound a eukaryote ribosome at the same helix 44, forcing the flipped-out orientation of two evolutionarily conserved adenine residues (A1492 and A1493) [37]. Actually, both molecules share rings I and II (2-deoxystreptamine ring common to all aminoglycosides), which are similarly anchored to the pocket core with ring I. This provides a structural explanation for our results indicating that the same amino acids are found in the presence of paromomycin or in the presence of G418 [17].

While similar amino acids were incorporated, their relative ratio can change depending on the cell type, the nucleotide environment, the drug used, and the patient $[17,33]$. This clearly raises the question of the major recoded protein produced. This issue still needs to be addressed for each single PTC, according to 
treatment and cell type. Nonetheless, in all cases, the incorporated amino acids correspond to the use of a near-cognate tRNA presenting a single mismatch in the first or third position (table S9). Although it is important to highlight that nucleotides surrounding the PTC could modify the identity of tRNAs inserted at the PTC [33], it seems unlikely that very different amino acids could be incorporated, due to the energetic and geometrical constraints to stabilise the codon:anticodon in a minihelix conformation [38] needed to catch the tRNA in the ribosome.

Recoding into a nonfunctional channel will reduce treatment efficacy, even if high levels of proteins can be produced. In the subset of 15 studied PTCs, seven PTCs were found to be recoded into at least one defective channel. This could be overcome with complementary CFTR-dedicated corrector/potentiator treatments. The activity of all channels was found to be increased by the CFTR potentiator VX770, a molecule already given with success to CF patients carrying gating-defective channels [39], while channels presenting a processing defect responded to the corrector/potentiator combined treatment (VX809/ VX770), a combination developed for the processing-defective p.Phe508del mutation [40]. Therefore, combining readthrough-inducing molecules and CFTR modulators should increase treatment efficacy, especially when the produced channels are defective. We provide evidence for correction of CFTR activity in primary nasal epithelial cells of a p.Gly $542^{*} / \mathrm{p}$.Gly542* homozygous patient. This mutation is the most frequent CF-causing PTC and is predicted to produce a misfolded channel (table 1). Gentamicin treatment induced a moderate improvement of cAMP-dependent chloride transport, which was significantly improved by the CFTR corrector. Similar results were also recently reported after G418 treatment in nasal epithelial cells of a patient with the same genotype [33] and in intestinal organoids from a patient carrying the p.Glu60* mutation [41], a mutation also predicted to be recoded into a misprocessed channel (table 1).

The combination of these in vitro results was used to classify the different PTCs with regard to readthrough treatment sensitivity. Nonetheless, we are aware that our in vitro assays have limitations. First, we could not take into account the level of NMD as this parameter, found to be very variable from one individual to another, needs to be measured for each patient [13]. Also, while in vitro assays measure the different defects (NMD, exon skipping and PTC readthrough) associated with PTCs independently, they do not reflect the state of primary cells where they occur simultaneously. Most importantly, the assays were performed in various cell lines, but obviously this could differ from native epithelium. Therefore, the translatability of drug-induced CFTR readthrough drugs from CF heterologous expression systems to CF primary cells is difficult to assess. Nonetheless, our classification is supported by preclinical studies, which showed that patients carrying PTCs predicted to be moderately or highly responsive to treatment responded on average with a higher frequency compared to PTCs predicted to be low responders (table 1). Therefore, the proposed classification can explain part of the variability observed in these eight studies.

Finally, readthrough therapy targets a molecular defect common to many genetic diseases and could therefore treat a substantial proportion of patients [1]. Studies have been performed in both in vitro models and clinical trials, showing an important variability in the response (reviewed in [4]). Nucleotide context, exon skipping and NMD potency, as well as the activity of the recoded protein, could in part explain these variabilities. Deciphering the molecular mechanism of each PTC for any given readthrough compound might enable the prediction of the outcome of treatment in the context of personalised therapy. Such data will allow the identification of the limiting step and the adaptation of the treatment with more efficient molecules or co-therapies, such as the ones available for CFTR, to optimise precision medicine.

\section{Acknowledgements}

L.J. Galietta (Telethon Institute of Genetics and Medicine (TIGEM), Naples, Italy) is acknowledged for kindly providing the halide-sensitive YFP construct, and R. Matsuda (Dept of Life Sciences, Graduate School of Arts and Sciences, The University of Tokyo, Tokyo, Japan) for providing the negamycin. This work benefited from the facilities and expertise of the SICaPS proteomic platform of I2BC (Institute for Integrative Biology of the Cell (I2BC), CEA, CNRS, Universite Paris-Sud, Université Paris-Saclay, Gif-sur-Yvette, France) for the mass spectrometry analysis.

\section{References}

1 Kellermayer R. Translational readthrough induction of pathogenic nonsense mutations. Eur J Med Genet 2006; 49: $445-450$.

2 Maquat LE. When cells stop making sense: effects of nonsense codons on RNA metabolism in vertebrate cells. RNA 1995; 1: 453-465.

3 Sterne-Weiler T, Howard J, Mort M, et al. Loss of exon identity is a common mechanism of human inherited disease. Genome Res 2011; 21: 1563-1571.

4 Lee HL, Dougherty JP. Pharmaceutical therapies to recode nonsense mutations in inherited diseases. Pharmacol Ther 2012; 136: 227-266.

5 Sermet-Gaudelus I, Renouil M, Fajac A, et al. In vitro prediction of stop-codon suppression by intravenous gentamicin in patients with cystic fibrosis: a pilot study. BMC Med 2007; 5: 5.

6 Wilschanski M, Yahav Y, Yaacov Y, et al. Gentamicin-induced correction of CFTR function in patients with cystic fibrosis and CFTR stop mutations. N Engl J Med 2003; 349: 1433-1441. 
7 Welch EM, Barton ER, Zhuo J, et al. PTC124 targets genetic disorders caused by nonsense mutations. Nature 2007; 447: 87-91.

8 Du L, Damoiseaux R, Nahas S, et al. Nonaminoglycoside compounds induce readthrough of nonsense mutations. J Exp Med 2009; 206: 2285-2297.

9 Mutyam V, Du M, Xue X, et al. Discovery of clinically approved agents that promote suppression of cystic fibrosis transmembrane conductance regulator nonsense mutations. Am J Respir Crit Care Med 2016; 194: 1092-1103.

10 Gonzalez-Hilarion S, Beghyn T, Jia J, et al. Rescue of nonsense mutations by amlexanox in human cells. Orphanet J Rare Dis 2012; 7: 58.

11 Howard MT, Shirts BH, Petros LM, et al. Sequence specificity of aminoglycoside-induced stop codon readthrough: potential implications for treatment of Duchenne muscular dystrophy. Ann Neurol 2000; 48: 164-169.

12 Keeling KM, Bedwell DM. Clinically relevant aminoglycosides can suppress disease-associated premature stop mutations in the IDUA and P53 cDNAs in a mammalian translation system. J Mol Med 2002; 80: 367-376.

13 Linde L, Boelz S, Nissim-Rafinia M, et al. Nonsense-mediated mRNA decay affects nonsense transcript levels and governs response of cystic fibrosis patients to gentamicin. J Clin Invest 2007; 117: 683-692.

14 Holbrook JA, Neu-Yilik G, Hentze MW, et al. Nonsense-mediated decay approaches the clinic. Nat Genet 2004; 36: 801-808.

15 Hinzpeter A, Aissat A, de Becdelievre A, et al. Alternative splicing of in-frame exon associated with premature termination codons: implications for readthrough therapies. Hum Mutat 2013; 34: 287-291.

16 Blanchet S, Cornu D, Argentini M, et al. New insights into the incorporation of natural suppressor tRNAs at stop codons in Saccharomyces cerevisiae. Nucleic Acids Res 2014; 42: 10061-10072.

17 Roy B, Friesen WJ, Tomizawa Y, et al. Ataluren stimulates ribosomal selection of near-cognate tRNAs to promote nonsense suppression. Proc Natl Acad Sci USA 2016; 113: 12508-12513.

18 Wilschanski M, Miller LL, Shoseyov D, et al. Chronic ataluren (PTC124) treatment of nonsense mutation cystic fibrosis. Eur Respir J 2011; 38: 59-69.

19 Sermet-Gaudelus I, Boeck KD, Casimir GJ, et al. Ataluren (PTC124) induces cystic fibrosis transmembrane conductance regulator protein expression and activity in children with nonsense mutation cystic fibrosis. Am J Respir Crit Care Med 2010; 182: 1262-1272.

20 Clancy JP, Bebok Z, Ruiz F, et al. Evidence that systemic gentamicin suppresses premature stop mutations in patients with cystic fibrosis. Am J Respir Crit Care Med 2001; 163: 1683-1692.

21 Clancy JP, Rowe SM, Bebok Z, et al. No detectable improvements in cystic fibrosis transmembrane conductance regulator by nasal aminoglycosides in patients with cystic fibrosis with stop mutations. Am J Respir Cell Mol Biol 2007; 37: 57-66.

22 Kerem E, Hirawat S, Armoni S, et al. Effectiveness of PTC124 treatment of cystic fibrosis caused by nonsense mutations: a prospective phase II trial. Lancet 2008; 372: 719-727.

23 Wilschanski M, Famini C, Blau H, et al. A pilot study of the effect of gentamicin on nasal potential difference measurements in cystic fibrosis patients carrying stop mutations. Am J Respir Crit Care Med 2000; 161: 860-865.

24 Floquet C, Hatin I, Rousset JP, et al. Statistical analysis of readthrough levels for nonsense mutations in mammalian cells reveals a major determinant of response to gentamicin. PLoS Genet 2012; 8: e1002608.

25 Aissat A, de Becdelievre A, Golmard L, et al. Combined computational-experimental analyses of CFTR exon strength uncover predictability of exon-skipping level. Hum Mutat 2013; 34: 873-881.

26 Hinzpeter A, Aissat A, Sondo E, et al. Alternative splicing at a NAGNAG acceptor site as a novel phenotype modifier. PLoS Genet 2010; 6: e1001153.

27 Fanen P, Clain J, Labarthe R, et al. Structure-function analysis of a double-mutant cystic fibrosis transmembrane conductance regulator protein occurring in disorders related to cystic fibrosis. FEBS Lett 1999; 452: 371-374.

28 Pranke IM, Hatton A, Simonin J, et al. Correction of CFTR function in nasal epithelial cells from cystic fibrosis patients predicts improvement of respiratory function by CFTR modulators. Sci Rep 2017; 7: 7375.

29 Suprynowicz FA, Upadhyay G, Krawczyk E, et al. Conditionally reprogrammed cells represent a stem-like state of adult epithelial cells. Proc Natl Acad Sci USA 2012; 109: 20035-20040.

30 Arakawa M, Shiozuka M, Nakayama Y, et al. Negamycin restores dystrophin expression in skeletal and cardiac muscles of $m d x$ mice. J Biochem 2003; 134: 751-758.

31 Aznarez I, Chan EM, Zielenski J, et al. Characterization of disease-associated mutations affecting an exonic splicing enhancer and two cryptic splice sites in exon 13 of the cystic fibrosis transmembrane conductance regulator gene. Hum Mol Genet 2003; 12: 2031-2040.

32 Hull J, Shackleton S, Harris A. Analysis of mutations and alternative splicing patterns in the CFTR gene using mRNA derived from nasal epithelial cells. Hum Mol Genet 1994; 3: 1141-1146.

33 Xue X, Mutyam V, Thakerar A, et al. Identification of the amino acids inserted during suppression of CFTR nonsense mutations and determination of their functional consequences. Hum Mol Genet 2017; 26: 3116-3129.

34 Mornon JP, Lehn P, Callebaut I. Atomic model of human cystic fibrosis transmembrane conductance regulator: membrane-spanning domains and coupling interfaces. Cell Mol Life Sci 2008; 65: 2594-2612.

35 Manuvakhova M, Keeling K, Bedwell DM. Aminoglycoside antibiotics mediate context-dependent suppression of termination codons in a mammalian translation system. RNA 2000; 6: 1044-1055.

36 Nissim-Rafinia M, Kerem B. Splicing modulation as a modifier of the CFTR function. Prog Mol Subcell Biol 2006; 44: $233-254$.

37 Shalev-Benami M, Zhang Y, Rozenberg $\mathrm{H}$, et al. Atomic resolution snapshot of Leishmania ribosome inhibition by the aminoglycoside paromomycin. Nat Commun 2017; 8: 1589.

38 Grosjean H, Westhof E. An integrated, structure- and energy-based view of the genetic code. Nucleic Acids Res 2016; 44: 8020-8040.

39 Accurso FJ, Rowe SM, Clancy JP, et al. Effect of VX-770 in persons with cystic fibrosis and the G551D-CFTR mutation. N Engl J Med 2010; 363: 1991-2003.

40 Wainwright CE, Elborn JS, Ramsey BW, et al. Lumacaftor-ivacaftor in patients with cystic fibrosis homozygous for Phe508del CFTR. N Engl J Med 2015; 373: 220-231.

41 Zomer-van Ommen DD, Vijftigschild LA, Kruisselbrink E, et al. Limited premature termination codon suppression by read-through agents in cystic fibrosis intestinal organoids. J Cyst Fibros 2016; 15: 158-162. 